\title{
DAMPING OF WAVES DUE TO A SOLUBLE FILM
}

\author{
K.K. PURI \\ Department of Mathematics \\ University of Maine \\ Orono, Maine 04469 \\ U.S.A. \\ (Received January 26, 1979)
}

ABSTRACT. The damping of gravity waves, forming on the surface of a layer of viscous, incompressible fluid which rests on top of a porous medium, is studied. It is assumed that the 'free' surface of the fluid has a thin film of soluble material adsorbed on it and the relaxation time for the equilibrium of the film is negligible in comparison to the free surface oscillations. The damping coefficient of the waves is calculated as a function of the parameters associated with the surfactant and the permeability.

KEY WORDS AND PHRASES. Waves in viscous Ziquids, Gravity waves, Damping of waves, Permeable beds.

2980 MATHEMATICS SUBJECT CLASSIFICATION CODES. 76505.

\section{INTRODUCTION.}

In a recent issue of this journal, the author published a paper (henceforth to be referred to as (I)), exploring the role of viscosity and permeability on the damping characteristics of a gravity wave [1]. The purpose of the present paper 
is to extend this analysis to include the effect of a soluble surface-active material and to examine afresh the damping of such waves. As before, we consider a two-layer model wherein a layer of viscous, incompressible fluid of finite thickness overlies an infinitely deep porous matrix which is assumed to be saturated with the same fluid. The system has a 'free' surface and an infinite horizontal extent.

That the presence of a surfactant such as oil on water has a significant effect on the damping of surface waves, is well known. As early as 1883, Aitken [2] gave an excellent qualitative physical explanation of the phenomenon and supported it by experimental studies. He attributed the calming effect of the oil to the variations of surface tension. Such variations, in the present context, are obtained when a disturbance propagating on the free surface of the fluid causes local and periodic changes in the distribution of the adsorbed material. The inhomogeneity thus developed gives rise to the surface tension gradient whose cangential component enables the contaminated fluid surface to support a tangential tress. The latter on a clean surface is zero, thus spelling out the hydrodynamlcal consequence of the presence of a surface-active substance.

The theoretical treatment of such problems has been carried out by several authors, viz., Dorrestein [3], Levich [4], Tempel and Riet [5], Miles [6], to mention a few. Dorrestein considers an insoluble film on the surface of an infinitely deep ocean but takes into account the surface viscosity and compressibility to discuss the damping of gravity waves in the frame work of a linearized theory. Levich works both with soluble as well as insoluble films. Tempel and Riet also attempt both the cases but they question the accuracy of Levich's boundary condition for the tangential stress. However, their own boundary condition seems to agree with that of Levich when appropriate comparisons are made [6]. Whereas the above authors formulate their problems in a body of fluid having infinite depth and 
infinite horizontal extent, Miles develops his analysis for surface waves in a closed basin.

The basic appeal in the present treatement is to waves in the ocean. Thus, whereas the fluid body is assumed unbounded, we adopt the viewpoint that in a realistic model there should be a porous medium underlying a finite layer of fluid. As such permeability is considered one of the possible mechanisms leading to the damping process.

\section{FORMULATION OF THE PROBLEM}

We consider a layer of viscous, imcompressible fluid of finite depth $h$ and infinite horizontal extent, overlying an infinitely deep porous bed which is saturated with the same fluid. Also we assume that the system is contaminated due to the presence of a soluble surface-active material having $c_{0}\left(\operatorname{moles} / \mathrm{cm}^{3}\right)$ and $\gamma_{0}$ (moles $/ \mathrm{cm}^{2}$ ) as the respective surfactant concentration and adsorbtion. The problem is to study the decay of a plane wave, viz., $\propto \exp \left(i \omega t^{\prime}-i k x^{\prime}\right)$ propogating in the direction of $x$ 'axis under the combined effect of the surface activity, the viscosity and the permeability. The $y^{\prime}$-axis is taken in the vertically upward direction.

The linearized Navier-Stokes equations of motion for the fluid and Darcy's equations for the bed are:

$$
\begin{aligned}
q_{1 t^{\prime}}^{\prime} & =-\frac{1}{\rho} \nabla^{\prime} p_{1}^{\prime}+\nu \nabla^{\prime}{ }^{\prime} q_{1}^{\prime} \\
\frac{1}{n} q_{2 t^{\prime}} & =-\frac{1}{\rho} \nabla^{\prime} p_{2}^{\prime}-\frac{D^{\prime}(n)}{\rho} q_{2}^{\prime}
\end{aligned}
$$

Here the subscripts ' $I$ ' and ' 2 ', used as ' $i$ ' below, refer to the fluid and the bed respectively. The quantities $\nabla^{\prime}=\left(\frac{\partial}{\partial x^{\prime}}, \frac{\partial}{\partial y^{\prime}}\right)$ and $q^{\prime}{ }_{i}=\left(u_{i}{ }^{\prime}, v_{i}{ }^{\prime}\right)$ represent the gradient and the velocity respectively and the latter satisfy the equations of continuity

$$
\nabla^{\prime} \cdot q_{1}^{\prime}=0 \text { and } \nabla \cdot \cdot q_{2}^{\prime}=0
$$

In the above formulation, the gravitational effect is included in the pressure term so as to write 


$$
P_{i}^{\prime}=P_{i}^{\prime}+\rho g y^{\prime}-P_{0}
$$

where, $\mathrm{P}^{\prime}{ }_{i}$ are the hydrodynamical pressures in the two regimes, $\mathrm{P}_{0}$ is the atmospheric pressure, $\rho$ is the fluid density and $g$ is the acceleration due to gravity. Also $\nu$ is the kinematic viscosity of the fluid, $n$ is the porosity of the medium and $D^{\prime}(n)$ represents the drag of the fluid due to the solid particles in the porous matrix. We assume that the particles are fixed so that $D^{\prime}(n)$ may be taken as a constant, equivalent to $\frac{\nu}{K^{\prime}}$, where $K^{\prime}$ is the bed permeability.

As mentioned earlier, the presence of a wave at the surface affects the distribution of both the bulk as well as the surface concentration of the contaminants. If these changes are from $c_{0}$ to $c_{0}+c^{\prime}\left(x^{\prime}, y^{\prime}, k^{\prime}\right)$ and $\gamma_{0}$ to $\gamma_{0}+\gamma^{\prime}\left(x^{\prime}, y^{\prime}, t^{\prime}\right)$, then, following Levich [4], we have,

$$
\frac{\partial c^{\prime}}{\partial t^{\prime}}=\lambda^{\prime} \nabla^{2} c^{\prime}
$$

where $\lambda^{\prime}$ is the diffusion constant of the surface material.

The equations (2.1) to $(2.5)$ are to be solved subject to the following boundary conditions:

the kinematic condition: $v^{\prime}{ }_{1}=E^{\prime}{ }_{t}$, at $y^{\prime}=0$

the vertical stress condition: $-P_{1}+2 \nu \rho v^{\prime}{ }_{1 y}{ }^{\prime}=\sigma^{\prime}{ }_{0}{ }^{\prime} x^{\prime} x^{\prime}$ at $y^{\prime}=0$

the horizontal stress condition: $\left.\rho v^{\prime} u^{\prime}{ }_{l y^{\prime}}+v^{\prime}{ }_{l x^{\prime}}\right)=\sigma^{\prime} x^{\prime}$ at $y^{\prime}=0$

Aside from the above, we also have the surface adsorption,

$\gamma_{0}+\gamma^{\prime}\left(x^{\prime}, y^{\prime}, t^{\prime}\right)$ governed by

$$
\gamma_{t^{\prime}}^{\prime}+\gamma_{0} u^{\prime} x^{\prime}+\lambda^{\prime} c^{\prime}{ }_{y^{\prime}}=0 \text { at } y=0
$$

Here we have used $E^{\prime}\left(x^{\prime}, t^{\prime}\right)$ for the wave elevation and $\sigma^{\prime}{ }_{0}+\sigma^{\prime}\left(x^{\prime}, y^{\prime}, t^{\prime}\right)$ for the variable surface tension, where $\sigma_{0}^{\prime}$ is the equilibrium value and $\sigma^{\prime}$ is the small perturbation consequent upon the wave motion. At the interface, we have

$$
\begin{gathered}
v_{1}^{\prime}=v_{2}^{\prime} \text { at } y^{\prime}=-h \\
u_{1 y^{\prime}}=\frac{\alpha}{\sqrt{K^{\prime}}}\left(u_{1}^{\prime}+\frac{1}{\rho D^{\prime}} P^{\prime} x_{2}\right) \text { at } y^{\prime}=-h \\
-P_{1}^{\prime}+2 v \rho v^{\prime}{ }_{1 y^{\prime}}=-P_{2}^{\prime} \text { at } y^{\prime}=-h
\end{gathered}
$$


The dimensionless number $\alpha$ is the slip constant. The conditions (2.10)-(2.12) were discussed in (I). Finally we have:

$$
\mathrm{q}^{\prime}{ }_{2} \rightarrow 0 \text { as } \mathrm{y}^{\prime} \rightarrow-\infty
$$

We now introduced the dimensionless variables based upon the wave number $k$ and the frequency $\omega$ of the wave motion. Thus we write:

$$
\begin{aligned}
(x, y) & =k\left(x^{\prime}, y^{\prime}\right), t=\omega t^{\prime},\left(u_{j}, v_{j}\right)=\frac{k}{\omega}\left(u^{\prime} j_{j}, v^{\prime}{ }_{j}\right), \\
P_{j} & =\left(k^{2} / \omega^{2}\right) \rho^{-1} p^{\prime} j, \quad F=g k / \omega^{2}, \quad \varepsilon=k^{2} v / \omega, \\
D & =D^{\prime} / \rho \omega, \quad K=k^{2} K^{\prime}, \quad E=E^{\prime} k, \quad \lambda=\lambda^{\prime} k^{2} / \omega, \quad \sigma=k^{3} \sigma^{\prime} / \rho \omega^{2}, \\
c^{\prime} & =c_{0} c, \quad \gamma^{\prime}=\gamma_{0} \gamma, \quad \eta=\alpha / \sqrt{k}
\end{aligned}
$$

Thus the equilibrium values of the dimensionless bulk concentration and the surface adsorption is each equal to 1 unit. We also define the dimensionless stream function $\psi_{j}$ by the usual relation, $\vec{q}_{j}=\nabla x\left(0,0, \psi_{j}\right)$. The above equations in terms of the new variables are:

$$
\begin{aligned}
& \left(\frac{\partial}{\partial t}-\varepsilon \nabla^{2}\right) \nabla^{2} \psi_{1}=0 \\
& \left(\frac{\partial}{\partial t}+n D\right) \nabla^{2} \psi_{2}=0 \\
& c_{t}=\lambda \nabla^{2} c \\
& E_{t}+\psi_{1 x}=0 \text { at } y=0 \\
& \varepsilon\left(\psi_{1 y y}-\psi_{1 x x}\right)=\sigma_{x} \text { at } y=0 \\
& \psi_{\text {lytt }}-\varepsilon\left(3 \psi_{\text {lyxxt }}+\psi_{\text {lyyyt }}\right)-F \psi_{1 x x}=-\sigma_{0} \psi_{1 x x x x} \text { at } y=0 \\
& \gamma_{t}+\psi_{1 y x}+\frac{\lambda c_{0}}{k \gamma_{0}} c_{y} \text { at } y=0 \\
& \psi_{1 \mathrm{x}}=\psi_{2 \mathrm{x}} \text { at } \mathrm{y}=-\mathrm{kh} \\
& (i+3 \varepsilon) \psi_{1 y}-\varepsilon \psi_{1 y y y}=\left(\frac{i}{n}+D\right) \psi_{2 y} \text { at } y=-k h \\
& \psi_{1 y y}=n\left(\psi_{1 y}-\psi_{2 y}-\frac{1}{n D} \psi_{2 y t}\right) \text { at } y=-k h \\
& \text { c, } \psi_{2} \rightarrow 0 \text { as } \mathrm{y} \rightarrow-\infty
\end{aligned}
$$


In order to find $\sigma$, we assume that the local equilibrium between the surface and the subsurface solution is constantly maintained. This amounts to saying that the relaxation time for the equilibrium between the material in the adsorbed film and the dissolved material in the underlying liquid is negligible compared with the period of the gravity waves. The experimental support for this assertion comes from the work of Davies and Vose [7]. Under this assumption, the surface tension $\sigma^{\prime}{ }_{0}{ }^{\prime} \sigma^{\prime}$ as well as the surfactant adsorption $\gamma_{0}+\gamma^{\prime}$ are completely determined by the surfactant concentration $c^{\prime}\left(x^{\prime}, E^{\prime}, t^{\prime}\right)=c_{0}+c^{\prime}\left(x^{\prime}, E^{\prime}, t^{\prime}\right) \simeq c_{0}+c^{\prime}\left(x^{\prime}, 0, t^{\prime}\right)$. As a result, we can write

$$
\sigma^{\prime}=c^{\prime}\left(x^{\prime}, 0, K^{\prime}\right)\left(\frac{d \sigma^{\prime}}{d c^{\prime}}\right)_{0}
$$

and

$$
\gamma^{\prime}=c^{\prime}\left(x^{\prime}, 0, t^{\prime}\right)\left(\frac{d^{\prime}}{d c^{\prime}}\right)_{0}
$$

where the subscript ' $O$ ' refers to the equilibrium value.

It has been shown by Adam [8] that the surface of a surfactant solution behaves, in many cases, like an ideal two dimensional solution, and in that case the adsorption is related to the concentration by means of a Langmuir adsorption isotherm

$$
\gamma_{0}+\gamma^{\prime}=\gamma_{\infty}^{\prime} \frac{c_{s}^{\prime}}{a^{\prime}+c_{s}^{\prime}}
$$

where $\gamma_{\infty}^{\prime}$ is the saturation adsorption in moles $/ \mathrm{cm}^{2}, a^{\prime}$ is szyszkowski constant which is that value of the surface concentration, $c^{\prime}{ }_{s}$ at which $\gamma_{0}+\gamma^{\prime}$ attains half its saturation value. Using (2.28) in (2.27) we get,

$$
\gamma^{\prime}=\frac{c^{\prime}\left(x^{\prime}, 0, t^{\prime}\right) a^{\prime} \gamma_{\infty}^{\prime}}{\left(a^{\prime}+c_{0}\right)^{2}}
$$

Also, under these conditions, the surface-tension depends upon the concentration as given by Szyszkowski's equation,

$$
\sigma_{\omega}^{\prime}-\left(\sigma^{\prime}+\sigma^{\prime}{ }_{0}\right)=R \theta \gamma_{\infty}^{\prime} \ln \left(1+\frac{c^{\prime} s}{a^{\prime}}\right)
$$


where $\sigma^{\prime}{ }_{\omega}$ is the surface tension of pure water, $R$ is the gas constant and $\theta$ is the absolute temperature.

$$
-\left.\frac{d \sigma^{\prime}}{d c^{\prime}}\right|_{0} \simeq \frac{R \theta \gamma_{\infty}^{\prime}}{\left(a^{\prime}+c_{0}\right)}
$$

Using the above equation in (2.26), we get

$$
\sigma^{\prime} \simeq-c^{\prime}\left(x^{\prime}, o, t^{\prime}\right) \frac{R \theta \gamma_{\infty}^{\prime}}{\left(a^{\prime}+c_{0}\right)}
$$

Writing the equations (2.29) and (2.32) in the dimensionless forms, we have

$$
\gamma=\frac{c(x, o, t) \gamma_{\infty}}{(a+1)^{2}}
$$

and

$$
\sigma=-\frac{k^{3} c(x, 0, t) R \theta \gamma_{\infty} \gamma_{0}}{\rho \omega^{2}(a+1)}
$$

Also from (2.30), we have for the equilibrium value,

$$
\sigma_{0} \simeq \sigma_{\omega}-\frac{\mathrm{RT} \gamma_{0} \gamma_{\infty} k^{3}}{\rho \omega^{2}} \ln \left(1+\frac{1}{a}\right)
$$

\section{SOLUTION OF THE PROBLEM}

Our interest is to discuss the decay of the plane wave solutions of the form $e^{i(t-x)}$ of the equations (2.15) to (2.17) subject to the auxiliary conditions (2.18) to $(2.25)$. Thus, for various unknowns in these equations, we write, as an example

$$
f(x, y, t)=F(y) e^{i(t-x)}
$$

and solve the resulting ordinary differential equations. The equations (2.15) and (2.16) with (2.25) yield,

$$
\begin{gathered}
\psi_{1}=\bar{A} e^{y+k h}+\bar{B} e^{-(y+k h)}+\bar{C} e^{-\beta(y+k h)}+\bar{D} e^{\beta y} \\
\psi_{2}=\bar{E} e^{y+k h}, \text { where } \beta^{2}=1+i / \varepsilon
\end{gathered}
$$

Assuming that $\lambda<<\varepsilon$, implying thereby that the thickness of the diffusion boundary layer is small compared to that of the viscous boundary layer, we have, from (2.17), 


$$
C=\bar{G} e^{\mu y}, \mu^{2}=1+i / \lambda
$$

where we have to take the branch of $\beta$ and $\mu$ on which $\sqrt{1}=1$. Substituting (3.1) - (3.3) in the equations (2.19) - (2.24) and invoking the relations (2.33), (2.34) for $\sigma$ and $\gamma$ we have:

$$
\begin{aligned}
& \varepsilon\left[2 \bar{A} e^{k h}+2 \bar{B} e^{-k h}+\bar{D}\left(1+\beta^{2}\right)\right]-\frac{i R T \bar{G} k^{3} \gamma_{0} \gamma_{\infty}}{\rho \omega^{2}(a+1)}=0 \\
& (1-2 i \varepsilon-L) \bar{A} e^{k h}-\bar{B} e^{-k h}(1-2 i \varepsilon+L)-\bar{D}(2 i \varepsilon \beta+L)=0 \\
& \bar{A} e^{k h}-\bar{B} e^{-k h}+\bar{D} \beta+\bar{G} M=0 \\
& \bar{A}+\bar{B}+\bar{C}-\bar{E}=0 \\
& \bar{A}(i+2 \varepsilon)-\bar{B}(i+2 \varepsilon)-2 \beta \varepsilon \bar{C}-D N \bar{E}=0 \\
& \bar{A}\left(\frac{1}{n}-1\right)+\bar{B}\left(\frac{1}{n}+1\right)+\bar{C}\left(\frac{\beta^{2}}{n}+\beta\right)+\bar{E} N=0 \\
& \text { where, L }=F+\sigma_{\omega}-\frac{R \theta k \gamma_{0} \gamma_{\infty}}{\rho \omega^{2}} \ln \left(1+\frac{1}{a}\right) \\
& M=\frac{i \lambda c_{0} \mu}{\gamma_{0}^{k}}-\frac{\gamma_{\infty} a}{(a+1)^{2}} \\
& N=1+\frac{i}{n D}
\end{aligned}
$$

Eliminating the 6 unknowns $\bar{A}, \bar{B}$ etc. from the above 6 equations we get the determinant (with some rows interchanged),

$\left|\begin{array}{llllll}1 & 1 & 1 & 0 & -1 & 0 \\ i+2 \varepsilon & -(i+2 \varepsilon) & -2 \varepsilon \beta & 0 & -D N & 0 \\ \varepsilon\left(\frac{1}{n}-1\right) & \varepsilon\left(\frac{1}{n}+1\right) & \varepsilon\left(\frac{\beta^{2}}{n}+\beta\right) & 0 & \varepsilon N & 0 \\ e^{k h} & -e^{-k h} & 0 & \beta & 0 & M \\ 2 \varepsilon e^{k h} & 2 \varepsilon e^{-k h} & 0 & \varepsilon\left(1+\beta^{2}\right) & 0 & \frac{-i R \theta \gamma_{0} \gamma_{\infty} k^{3}}{\rho \omega^{2}(a+1)} \\ e^{k h}(1-2 i \varepsilon-L) & -e^{-k h}(1-2 i \varepsilon+L) & 0 & -(2 i \varepsilon \beta+L) & 0 & 0\end{array}\right|=0$

Expanding it in powers of $\varepsilon^{1 / 2}$, we have, 


$$
\begin{aligned}
L\left[\frac{1}{\eta}(i+N D T)+\right. & \left.\sqrt{i} \sqrt{\varepsilon}(1-i N D T)+\varepsilon\left(i N D+N+\frac{3}{\eta}\right)+\sqrt{i} \varepsilon^{3 / 2}\left(2 i N T-\frac{1}{2} N D T+\frac{2 i T}{\eta}+\frac{i}{2}\right)\right] \\
& -\frac{1}{\eta}(i T+N D)+\sqrt{i} \sqrt{\varepsilon}(i N D-T)-\varepsilon\left(i T D N+T N+\frac{7 T}{\eta}-\frac{4 i N D}{\eta}\right) \\
& -\sqrt{i} \varepsilon^{3 / 2}\left(2 i N-\frac{9}{2} N D+\frac{6 i}{\eta}-\frac{9}{2} i T+4 N D T\right)+\bar{M}\left[L \left\{\frac{\sqrt{i}}{\sqrt{\varepsilon}}(1-i N D T)+(1-i N D T)\right.\right. \\
& +\frac{1}{n}(-T+i N D)+\sqrt{i} \sqrt{\varepsilon}\left(2 D N-i N-\frac{7 i}{2 \eta}-\frac{N D T}{2 \eta}+\varepsilon\left(3 i N T-2 N D T+\frac{5 i T}{\eta}\right)\right. \\
& \left.-\sqrt{i} \varepsilon^{3 / 2}\left(\frac{9 N}{2}+\frac{43^{0}}{8 \eta}+i D N-\frac{T}{2}+\frac{i N D T}{8 \eta}\right)\right\} \\
& +\frac{\sqrt{i}}{\sqrt{\varepsilon} \eta}(i D N-T)+(i D N-T)+\sqrt{i} \sqrt{\varepsilon}\left(-T N D+T i N+\frac{7 i T}{2 \eta}+\frac{N D}{2 \eta}\right) \\
& \left.+\varepsilon\left(5 N D-2 i N+i T-\frac{2 i}{\eta}\right)+\sqrt{i} \varepsilon^{3 / 2}\left(\frac{129}{8} \frac{T}{\eta}+\frac{i D N T}{2}+\frac{5 N T}{2}+\frac{D i N}{8 \eta}\right)\right]+0\left(\varepsilon^{2}\right)=0 \\
& \text { where } \bar{M}=\frac{c R \theta \gamma \gamma_{0} k^{3}}{\rho \omega^{2} M(a+1)} \\
& \text { and } T=\operatorname{tanh(kh).}
\end{aligned}
$$

The equation (3.8) together with (2.14) yields the dispersion relation giving the frequency $\omega$ of the propogating wave as a function of the physical parameters. In order to find its antecedent, we set $\varepsilon=\bar{M}=0$ in the above equation. Denoting $\omega$ by $\omega_{\circ}$ in this case, we have,

$$
\left(g k+\frac{k^{3} \sigma_{0}^{\prime}}{\rho}\right)\left\{i+T\left(D+\frac{i}{n}\right)\right\}=\omega_{0}^{2}\left(i T+D+\frac{i}{n}\right) .
$$

Using it in the dimensional variables from (2.14) and separating the resulting equation into the real and the imaginary parts, we get

$$
\omega_{\circ}^{2}=\left(g k+\frac{k^{3} \sigma_{0}^{\prime}}{\rho}\right) \tanh k h
$$

and

$$
\omega_{\circ}^{2}=\left(g k+\frac{k^{3} \sigma_{\circ}^{\prime}}{\rho}\right)\left(\frac{\tanh k h+n}{1+n \tanh k h}\right)
$$

giving us the two possible modes of vibration. This result was established by Puri in an earlier paper [9]. 
Also if we set $\bar{M}=0$, the equation (3.8) reduces to its analogue in (I). 4. DISCUSSION OF THE SOLUTION.

The principal objective is to study the damping characteristics of the wave form $\exp \left(i \omega t^{\prime}-i k x^{\prime}\right)$ as it evolves from an initially assumed form $\exp \left(i \omega_{0} t^{\prime}-i k x^{\prime}\right)$, where $\omega_{0}$ is given by (3.10). If $\omega=\omega_{0}+\omega_{1},|\omega| \ll \omega_{0}$, then the condition that the wave will be damped out is $\operatorname{Im} \omega_{1}>0$. To find $\omega_{1}$, on the other hand, we invoke the equation (3.8) subject to (2.14) and obtain

$$
\begin{aligned}
& \frac{\omega_{1}}{\omega_{0}}\left[\frac{P \sqrt{i}}{n \sqrt{\varepsilon_{0}}}\left\{2\left(\frac{1}{N_{0} D_{0}}-i T\right)+\frac{2 Q\left(1-T^{2}\right)}{N_{0} D_{0}}-\frac{\left(1-T^{2}\right)}{2 N_{0} D_{0}}\right\}+\frac{2 P Q\left(1-T^{2}\right)}{N_{0} D_{0}}\right. \\
& -\frac{P}{\eta}\left(\frac{2 Q T}{N_{0} D_{0}}-2 i Q-\frac{i}{N_{0}}-\frac{2}{N_{0} D_{0}}+2 i T-2 i+\frac{2 T}{N_{0} D_{0}}\right)+\frac{2}{n}\left(T+\frac{i}{N_{O} D_{0}}\right) \\
& +\sqrt{i} \sqrt{\varepsilon_{0}}\left\{P\left(2 Q+\frac{1}{2}\right)\left(\left(2-T^{2}\right)-\frac{i\left(1-T^{2}\right)}{D_{0}}-\frac{7 i\left(1-T^{2}\right)}{2 n N_{0} D_{0}}+\frac{i T}{N_{0} D_{0}}\right)\right. \\
& \left.\left.+P\left(\frac{\left(2-T^{2}\right)}{N_{0}}-\frac{\left(1-T^{2}\right)}{2 n N_{0} D_{0}}+\frac{\left(1-T^{2}\right)}{2 N_{0} D_{0}}+4-\frac{2 i}{D_{0}}-\frac{14 i}{2 n N_{0} D_{0}}+\frac{2 i T}{N_{0} D_{0}}-\frac{T}{\eta}\right)-2 i T+\frac{2}{N_{0} D_{0}}\right)\right\} \\
& =P\left[\frac{\sqrt{i}\left(1-T^{2}\right)}{\sqrt{\varepsilon_{0}} n N_{0} D_{0}}+\frac{\left(1-T^{2}\right)}{N_{0} D_{0}}+\frac{1}{\eta}\left(i-\frac{T}{N_{0} D_{0}}\right)\right]+\frac{i\left(1-T^{2}\right)}{n N_{0} D_{0}}+\sqrt{i} \sqrt{\varepsilon_{0}} \\
& {\left[P\left\{\left(2-T^{2}\right)-\frac{i\left(1-T^{2}\right)}{D_{0}}-\frac{7 i\left(1-T^{2}\right)}{2 \eta N_{0} D_{0}}+\frac{i T}{N_{0} D_{0}}\right\}+\frac{\left(1-T^{2}\right)}{N_{0} D_{0}}\right]} \\
& +\varepsilon_{0}\left[P\left(3 T+\frac{i T}{D_{0}}+\frac{3 i T}{\eta N_{0} D_{0}}+\frac{i T^{2}}{N_{0} D_{0}}\right)+i\left(1-T^{2}\right)+\frac{\left(1-T^{2}\right)}{D_{0}}+\frac{\left(3-7 T^{2}\right)}{\eta N_{0} D_{0}}+\frac{4 i T}{\eta}\right]+0\left(\varepsilon^{3 / 2}\right) \\
& \text { where } M_{0}=\frac{i^{3 / 2} c_{0} \sqrt{\lambda}}{k \gamma_{0}}-\frac{\gamma_{\infty} a}{(a+1)^{2}} \\
& P=\frac{i R \theta \gamma_{0} \gamma_{\infty} k^{3}}{\rho \omega_{0}^{2} M_{0}(a+1)}
\end{aligned}
$$

and 


$$
Q=1-\frac{i^{3 / 2} c_{0} \sqrt{\lambda_{0}}}{4 k \gamma_{0} n_{0}}
$$

The quantities with the subscript 0 are the same as their unsubscripted alanogues, scaled to the initial frequency $\omega_{0}$.

For a bed of sand, the permeability $\mathrm{K}^{\prime}=0\left(10^{-6}\right) \mathrm{cm}^{2}$. We assume that the kinematic viscosity is $0\left(10^{-2}\right) \mathrm{cm}^{2} / \mathrm{sec}$ and the water density $\rho$ is $1 \mathrm{gm} / \mathrm{cm}^{3}$ so that $D^{\prime}=0\left(10^{4}\right)$. Also, for water $\alpha=0(1)$, and, therefore, $n=0\left(10^{3}\right)$. The porosity $\mathrm{n}$ may be taken to be .35 so that $\mathrm{n}=\mathrm{N}_{\mathrm{o}}=0(1)$.

The values of the various surface activity parameters can vary vastly by several orders of magnitudes, depending upon the particular solute. The parameters $P, Q$, besides, depend upon $k$ and $\omega_{0}$. The numerical data used here is taken from the afore-mentioned work of Tempel and Riet. We shall denote it by DATA. It consists, in appropriate units, of $c_{0}$, varying between, $10^{-12}$ to $10^{-5}, \Gamma_{\infty}{ }^{\prime}$ between $10^{-10}$ and $10^{-9}$ and $a^{\prime}$ between $10^{-10}$ to $10^{-6}$. The frequency $\omega_{0}$ is calculated from the equation (3.10), for a depth $h$ of 5 to $20 \mathrm{cms}$ and a range of wave lengths varying from .5 to $6 \mathrm{cms}$ at intervals of $.5 \mathrm{cms}$. (The results mentioned below are for $\mathrm{h}=5 \mathrm{cms}$ only).

It turns out that for $a^{\prime}=O\left(10^{-6}\right)$ to $O\left(10^{-5}\right) \mathrm{cm}^{2}, \Gamma_{\infty}^{\prime}=0\left(10^{-10}\right)$ to $0\left(10^{-9}\right)$ and $c_{0}=10^{-9}$ to $10^{-8}$ moles $/ \mathrm{cm}^{3}, \gamma_{0}$ varies from $0\left(10^{-13}\right)$ to $0\left(10^{-11}\right)$ moles $/ \mathrm{cm}^{2}$ and $P$ assumes a value between $O\left(10^{-2}\right)$ to $O\left(10^{-1}\right)$. This holds for the full range of 12 wave lengths mentioned above. In this case, that is, taking $P=0\left(10^{-1}\right)$, the expression for the damping of $\omega_{1}$, simplifies considerably and becomes:

$$
\omega_{1}=\frac{\omega_{0}}{2 T}\left[i P\left(2-T^{2}\right)+i^{3 / 2} \sqrt{\varepsilon_{0}}\left(1-T^{2}\right)\right]
$$

whence from we can easily calculate,

$$
\operatorname{Im} \omega_{1}=\frac{\omega_{0}}{2 T}\left[\beta\left(2-T^{2}\right)+\sqrt{\varepsilon_{0}}\left(1-T^{2}\right) / \sqrt{2}\right],
$$


where $\quad \beta=\frac{\sqrt{2} R \theta c_{0} k^{4} \Gamma_{\infty}^{2}}{\rho \omega_{0}^{2} a^{\prime}} \frac{\left(a^{\prime} \sqrt{\lambda}+\sqrt{2} k \Gamma_{\infty}{ }^{\prime}\right)}{\left(a^{\prime} \sqrt{\lambda}\right)^{2}+\left(a^{\prime} \sqrt{\lambda}{ }_{0}+\sqrt{2} k \Gamma_{\infty}^{\prime}\right)^{2}}$

which shows that even a small amount of surface adsorption, that is, of the order $0\left(10^{-13}\right)$ can produce damping which is comparable to the viscous damping in the order of magnitude. The permeability in this case, however, is completely ineffective as a dissipative mechanism.

As mentioned in (I), there are situations in which the damping due to percolation may not be negligible. The wave motion in shallow water, for example, may markedly reduce the size of $D$ due to the upward flow of water. Also the drainage of the water table due to the outgoing tide could increase the porosity significantly. (These cases are discussed by Murray $[10,11]$. As a result, $K^{\prime}$ may be $O\left(15^{-4}\right)$ or $O\left(10^{-3}\right)$ so that $D^{\prime}=O\left(10^{2}\right)$ or $O(10)$ and $\eta=O\left(D^{\prime}\right)$.

Under these conditions, the equation (3.8), up to the first order, becomes:

$$
\begin{gathered}
\omega_{1}=\omega_{0} \frac{\text { Num }}{\text { Den }}, \text { where } \\
N u m=\frac{i P}{2 T}\left[\frac{\left(1-T^{2}\right)}{\sqrt{\varepsilon_{0} \eta N} D_{0}}+\frac{i^{-1 / 2}\left(1-T^{2}\right)}{N_{0} D_{0}}+\frac{\sqrt{i}}{\eta}+\sqrt{\varepsilon_{0}}\left(2-T^{2}\right)\right] \\
+\frac{i^{3 / 2}\left(1-T^{2}\right)}{2 T}\left(\frac{1}{\eta N_{0} D_{0}}+\varepsilon_{0}\right) \\
\text { DEN }=\frac{P}{n \sqrt{\varepsilon_{0}}}+\frac{\sqrt{i}}{\eta}+\sqrt{\varepsilon_{0}}
\end{gathered}
$$

As before, the quantity $P$ in $(4.9)$ and $(4.10)$ is taken to be $O\left(10^{-1}\right)$. Following the usual procedure, it can be shown that $\operatorname{Im} \omega_{1}>0$, thus leading to the decay of the wave. The table I below compares the values of Im $\omega_{1}$ based on the equations (4.6) and (4.8). It is easy to see the roll of the permeability. The interesting feature of the result (4.8) is that the contributions to the damping due to the presence of the three factors, viz., surface adsorption, viscosity and the 
permeability are not just additive but are also interactive. On the other hand, the full range of values of DATA produces $P$ as low as $0\left(10^{-19}\right)$. Obviously then the first four terms in (4.9) and the first term in (4.10) are irrelevant and therefore get discarded. The damping then is uneffected by the surface activity. This conclusion agrees with that of Miles [6].

A matter of interest in these problems is to study the variation of $\operatorname{Im} \omega_{1}$ as a function of the bulk concentration, $c_{0}$. This study was carried out on the bases of the complete equation (4.1) and the full range of DATA values. Table II lists the main results. It shows that the damping increases with $c_{0}$ until a certain maximum is reached; thereafter, the damping actually decreases. This agrees with the available theoretical studies of Tempel and Riet, and the experimental findings of Davies and Vose, Table I: Variations of $\operatorname{Im}\left(\omega_{1}\right)$ with and without Porosity

\begin{tabular}{c|l|l} 
Wave length $(\mathrm{cm})$ & \multicolumn{2}{|l}{$10^{3} \times \operatorname{Im}\left(\omega_{1}\right)$ based on } \\
\cline { 2 - 3 } .5 & equation 4.6 & equation 4.8 \\
\hline 1.0 & 0.827192 & 3.07999 \\
1.5 & 0.516942 & 1.32250 \\
2.0 & 0.388364 & 0.880049 \\
2.5 & 0.319839 & 0.691694 \\
3.0 & 0.277370 & 0.586376 \\
3.5 & 0.248289 & 0.515857 \\
4.0 & 0.226939 & 0.462615 \\
4.5 & 0.210458 & 0.419288 \\
5.0 & 0.197251 & 0.382454 \\
5.5 & 0.186364 & 0.350370 \\
6.0 & 0.177195 & 0.322064 \\
& & 0.296936
\end{tabular}




\section{REFERENCES}

1. Puri, K. K. Viscous Damping of Gravity Waves Over a Permeable Bed, Int. J. Math. \& Math. Sci. 1 (1978), 497-507.

2. Aitken, J. On The Effect of Oil on a Stormy Sea, Proc. Roy. Soc. Edinb. 12 (1883), 56-75.

3. Dorrestein, R. General Linearized Theory of the Effect of Surface Films on Water Ripples, Nederl. Akad. Van Wetenschapen B54 (1951), 250-272.

4. Levich, G. Physiocochemical Hydrodynamics, Prentice Hall, Englewood, N. J., 1962 .

5. Tempel, M. \& Riet, R. P. Damping of Waves by Surface Active Material, Journal Ch. Physics 8 (1965), 2769-2777.

6. Miles, J. W. Surface Wave Damping in Closed Basins, Proc. Roy. Soc. London Ser. A, 297 (1967) 459-475.

7. Davies, J. T. \& Vose, R. M. On the Damping of Capillary Waves by Surface Films, Proc. Roy. Soc. A 286 (1965) 218-230.

8. Adam, N. K. The Physics \& Chemistry of Surfaces, Dover Publications, New York, 1968.

9. Puri, K. K. Damping of Gravity Waves over a Porous Bed, to appear in ASCE Proceedings, J. of Hydraulics Division.

10. Murray, J. D. Viscous Damping of Gravity Waves, 70 (1965), 2325-2331.

11. Murray, J. D. On the Mathematics of Fluidization I, J. Fluid Mech. 21 (1965), 465-493. 


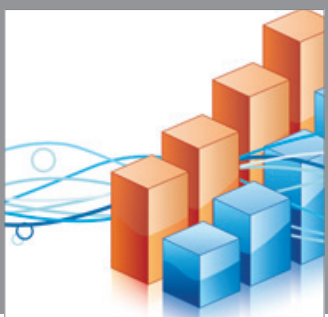

Advances in

Operations Research

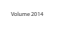

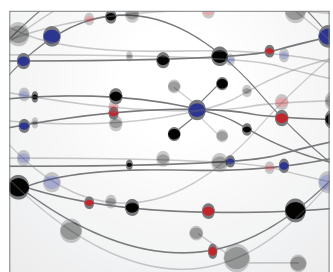

\section{The Scientific} World Journal
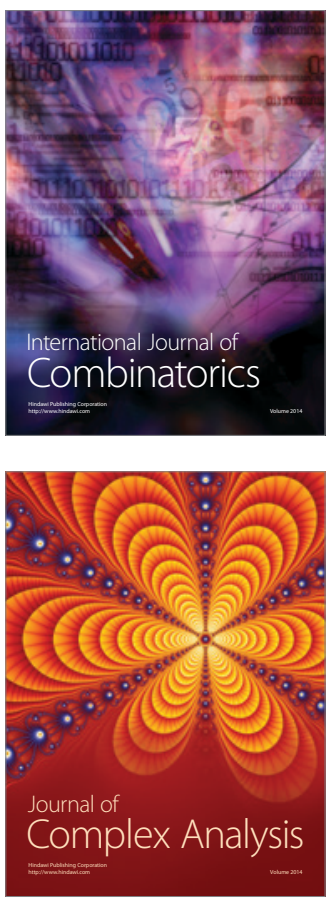

International Journal of

Mathematics and

Mathematical

Sciences
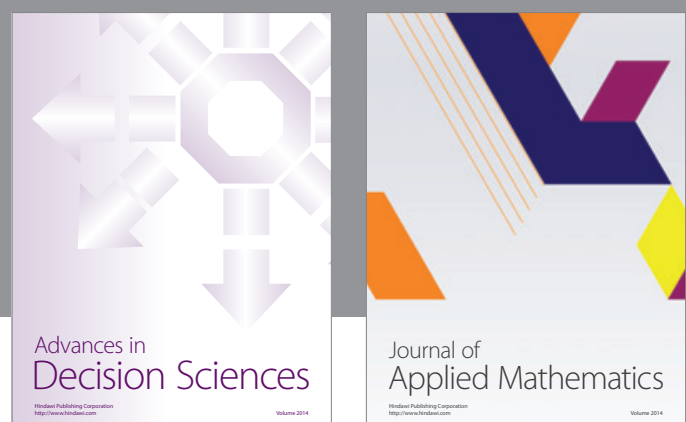

Journal of

Applied Mathematics
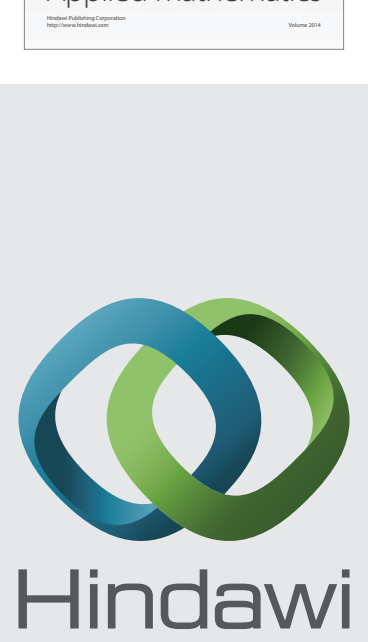

Submit your manuscripts at http://www.hindawi.com
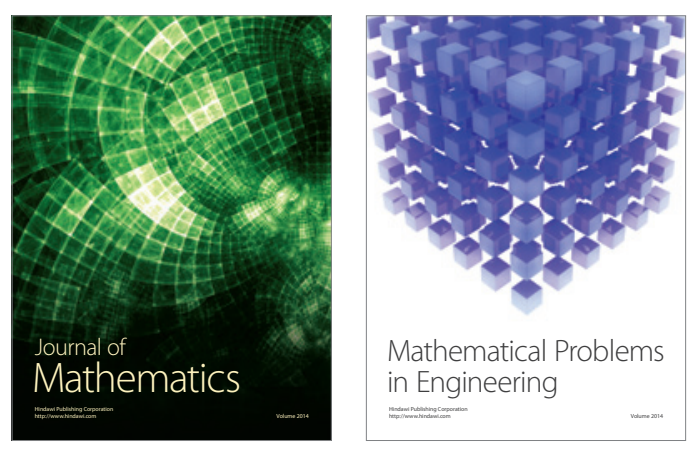

Mathematical Problems in Engineering
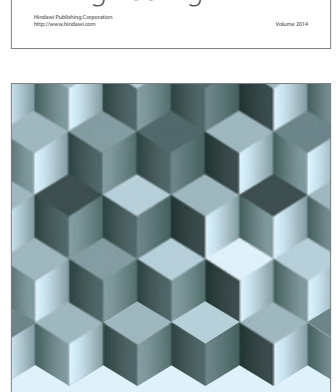

Journal of

Function Spaces
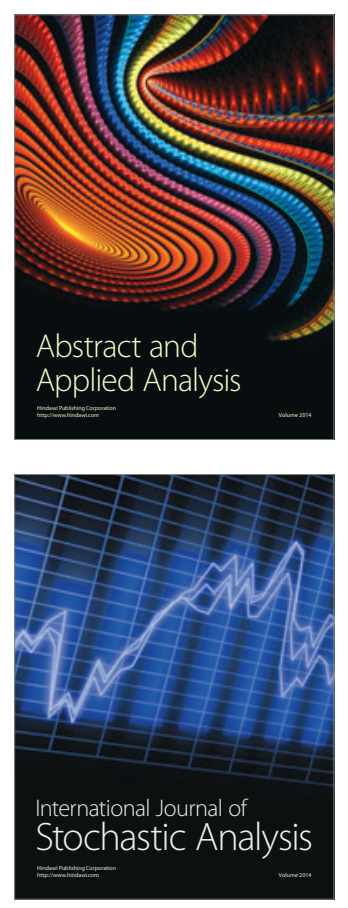

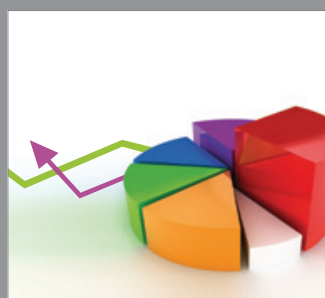

ournal of

Probability and Statistics

Promensencen
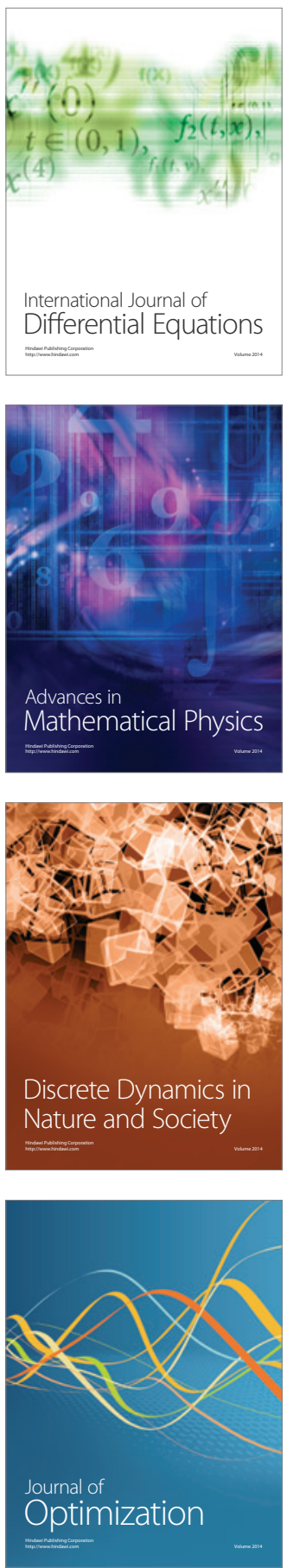